\title{
Comparison of insulin degludec (IDeg)/insulin Aspart (IAsp) co-formulation therapy twice-daily with free combination of GLP-1 receptor agonist liraglutide plus insulin deguldec in Tochigi: IDEAL Trial
}

\author{
Yoshimasa Aso ${ }^{1}$, Teruo Jojima ${ }^{2}$, Isao Usui ${ }^{2}$, Yoshihisa Takada ${ }^{3}$, Ken Tomotsune ${ }^{4}$, Yasuko \\ Chiba $^{5}$, Mihoko Matsumura ${ }^{6}$, Nobuya Fujita ${ }^{7}$, Hisamoto Kuroda ${ }^{8}$, Shunichi Murano ${ }^{9}$, and \\ Minoru Sato ${ }^{10}$ \\ ${ }^{1}$ Dokkyo Medical University \\ ${ }^{2}$ Dokkyo Ika Daigaku \\ ${ }^{3}$ Takada Clinic \\ ${ }^{4}$ Saiseikai Utsunomiya Byoin \\ ${ }^{5}$ Nagasaki Hospital \\ ${ }^{6}$ Kamitsuga General Hospital \\ ${ }^{7}$ Fujita Clinic \\ ${ }^{8}$ Green Clinic \\ ${ }^{9} \mathrm{TMC}$ Tochigi Medical Center \\ ${ }^{10}$ NHO Utsunomiya National Hospital
}

June 4, 2020

\begin{abstract}
Aim: We compared the efficacy and safety of insulin degludec/insulin aspart co-formulation (IDegAsp) twice-daily to a free combination of basal insulin degludec and GLP-1 receptor agonist liraglutide (IDeg+Lira) once-daily for patients with inadequately controlled type 2 diabetes on insulin therapy and oral antidiabetic drugs. Subjects and Methods: Eligible patients were randomly allocated at a 1:1 ratio to receive either the once-daily dual-injection of IDeg+Lira $(\mathrm{n}=24)$ or twice-daily singleinjection of IDegAsp $(n=28)$. The primary endpoints were: HbA1c changes over 52 weeks of treatment and the percentage of participants achieving HbA1c $<7.0 \%$ at week 52 . Results: After 52 weeks, HbA1c decreased by $0.3 \%$ in the IDegAsp group and by $0.7 \%$ in the IDeg+Lira group. The HbA1c reduction was greater in the IDeg+Lira group than in the IDegAsp group. $19 \%$ of patients on IDegAsp versus $40 \%$ on IDeg+Lira achieved HbA1c $<7.0 \%$. Pre-breakfast and pre-dinner blood glucose at 52 weeks were significantly lower in the IDeg+Lira group than in the IDegAsp group. The reduction in body mass index (BMI) was greater in the IDeg+Lira group than in the IDegAsp group throughout the study period. The confirmed hypoglycemia rates were 1.32 and 0.69 per patient/year of exposure to IDegAsp and IDeg+Lira, respectively. Conclusions: In patients with inadequately controlled type 2 diabetes on insulin therapy and oral antidiabetic drugs, treatment with the once-daily dual-injection of IDeg+Lira compared to the twice-daily single-injection of IDegAsp showed no significant difference in glycemic control, but with a slightly larger reduction in HbA1c at 52 weeks, and statistically superior weight loss.
\end{abstract}

\section{What is already known about this topic?}

Insulin degludec/insulin aspart (IDegAsp) is the first soluble co-formulation of a long-acting insulin analog (IDeg:70\%) and a rapid-acting insulin analog (IAsp:30\%). This co-formulation is easier for patients to 
administer because it does not require resuspension.

A meta-analysis determined that the combination of basal insulin and GLP-1 receptor agonist produces similar effects in patients as the effects of the intensified basal-bolus insulin regimens;

\section{What does this article add?}

In this randomized, active-controlled, open-label trial, we compared for the first time the efficacy and safety of IDegAsp twice-daily with the free combination of basal insulin degludec and GLP-1 receptor agonist liraglutide (Lira) once-daily in patients with type 2 diabetes on insulin therapy.

Treatment with the once-daily dual-injection of IDeg+Lira compared to the twice-daily single-injection of IDegAsp showed no significant difference in glycemic control, but with a slightly larger reduction in HbA1c at 52 weeks, and statistically superior weight loss.

\section{Introduction}

Due to the progressive nature of type 2 diabetes, the majority of diabetic patients eventually require insulin therapy (1). The addition of basal insulin to orally administered agents is a well-established approach and is effective for most patients (1). A basal insulin-only regimen has the advantage of being well-tolerated with frequent injection times and a low hypoglycemia risk, thereby facilitating patient acceptance of insulin therapy (2).

However, a basal insulin-only regimen is not always sufficient for maintaining proper glycemic control. The possible intensification options for maintaining postprandial glucose control are to either add a basal-bolus regimen, which involves taking a bolus of insulin at one to three meal times daily, or switch to a premixed insulin regimen twice-daily (1). Many patients with type 2 diabetes perceive the basal-bolus regimen as inconvenient and too complicated because it requires numerous daily injections and the administration of two different types of insulin (3).

Insulin degludec/insulin aspart (IDegAsp) is the first soluble co-formulation of a long-acting insulin analog (IDeg:70\%) and a rapid-acting insulin analog (IAsp:30\%). This co-formulation preserves the glucose-lowering effects of both fasting and prandial components; also, it is easier for patients to administer because it does not require resuspension (4). Several studies demonstrated that taking IDegAsp twice-daily more effectively improved glycemic control than did biphasic insulin aspart 30 because it can superiorly reduce fasting plasma glucose (FPG) levels and episodes of nocturnal hypoglycemia $(5,6)$. For many patients, the twicedaily IDegAsp regimen may provide a better solution than the basal-bolus regimen because this regimen of premixed insulin consists of only 2 injections.

Recently, the intensification guidelines recommended the combination of glucagon-like peptide-1 receptor agonists (GLP-1 RA) as an alternative to basal insulin. (7). A meta-analysis determined that the combination of basal insulin and GLP-1 RA produces similar effects in patients as the effects of the intensified basal-bolus insulin regimens; these similar effects include glucose-lowering, less weight gain, and hypoglycemia (8). A previous study showed that IDeg+Lira is superior to both basal insulin-only regimens and regimens of basal insulin + a single-dose of rapid insulin; patients took IDeg+Lira with the most substantial meal for the maximum glucose-lowering effects (9). Therefore, this combination is both effective and well-tolerated for the treatment of type 2 diabetes. However, no studies have yet to compare these two regimens in patients with inadequately controlled type 2 diabetes on insulin therapy. Thus, we are the first study to compare the efficacy and safety of IDegAsp twice-daily with the free combination of basal insulin degludec and GLP-1 receptor agonist liraglutide once-daily in patients with inadequately controlled type 2 diabetes on insulin therapy with or without additional oral antidiabetic drugs.

\section{Subjects and methods}

Patients were eligible to participate in this study if they met the following criteria: [?] 18 years old, body mass index $(\mathrm{BMI})<40.0 \mathrm{~kg} / \mathrm{m}^{2}$, inadequate glycemic control (HbA1c [?] $7.0 \%$ and $<10.5 \%$ ) for at least 3 months before screening, treatment with stable daily doses of insulin, and additional treatment with one-or-more 
oral antidiabetic drugs (metformin, sulfonylureas, glinides, dipeptidyl peptidase 4 inhibitors, sodium-glucose co-transporter 2 inhibitors, thiazolidinediones, or $\alpha$-glucosidase inhibitors).

This 52-week, randomized, controlled, open-label, multicenter, treat-to-target trial was carried out from December 2016 to December 2018; it compared the efficacy and safety of twice-daily single-injection of IDegAsp to the once-daily dual-injection of IDeg+Lira. Eligible patients were randomly allocated, by a 1:1 ratio, to receive IDegAsp twice-daily $(\mathrm{n}=29)$ or IDeg+Lira once-daily $(\mathrm{n}=28)$.

In the IDegAsp group, we reduced the starting dose of IDegAsp by $20 \%$ of the total daily-dose of insulin given at each screening. IDegAsp (70\% IDeg and 30\% IAsp, FlexTouch) was administered at breakfast and dinner, which split up the total daily dosage into two doses; however, the dosage of each dose varies, depending on the size of each meal. IDegAsp was titrated to achieve a target fasting blood glucose (FBG) $<110 \mathrm{mg} / \mathrm{dl}$ for the mean pre-breakfast glucose-value and the mean pre-dinner glucose-value, which were determined by patients that take self-measurements of blood glucose (SMBG). The dose was titrated once a week by patients. However, in fact, the titration of insulin dose was given discretion on each physician's decision.

In the IDeg+Lira group, the combination of IDeg (100U/mL, FlexTouch pen, Novo Nordisk A/S, Denmark) and liraglutide $(6 \mathrm{mg} / \mathrm{mL})$ once-daily were separately injected before breakfast. The starting dose of IDeg was equal to the dose of basal insulin administered at the screening. IDeg was titrated to achieve an FBG $<110 \mathrm{mg} / \mathrm{dl}$ on the mean pre-breakfast blood glucose value. Liraglutide was administered at breakfast, and the dose was increasingly titrated from $0.3 \mathrm{mg} /$ day to $0.6 \mathrm{mg} /$ day and then to $0.9 \mathrm{mg} /$ day over the 3 weeks. Titration to each new dosage required at least 1 week, but it could be delayed if the patient had severe nausea or vomiting at the investigator's discretion. Finally, all patients received a once-daily subcutaneous injection of $0.9 \mathrm{mg}$ of liraglutide and were followed for 52 weeks. As of May 22, 2019, the standard dose of liraglutide is $0.9 \mathrm{mg}$ in Japan. Dipeptidyl peptidase -4 (DPP-4) inhibitors were discontinued after the randomization step of the trial.

The primary endpoints were: HbA1c changes over 52 weeks of treatment and the percentage of participants achieving $\mathrm{HbA} 1 \mathrm{c}<7.0 \%$ at week 52 . Secondary endpoints included a 2-point daily SMBG profile taken before both breakfast and dinner (pre-breakfast and pre-dinner) at 12, 24, and 52 weeks; daily-dose of either insulin or liraglutide at 52 weeks; and changes in body weight or BMI at 52 weeks.

Confirmed hypoglycemic episodes were defined as detected plasma glucose level $<3.9 \mathrm{mmol} / \mathrm{L}(70 \mathrm{mg} / \mathrm{dL})$ regardless of the severity of either symptoms or episodes. We calculated the rate of hypoglycemic episodes per patient/year of exposure. Hypoglycemic episodes occurring from 0:00 and 05:59 were classified as nocturnal.

All participants gave written informed consent before enrollment. The institutional review board of Dokkyo Medical University Hospital approved this study. This study was registered with the University Hospital Medical Information Network Clinical Trials Registry (UMIN000024865).

\section{Statistical analysis}

No formal sample size calculations were made. Results are expressed as the mean \pm SD or as the median and IQR. Differences between groups were analyzed by Student's paired t-test or the unpaired t-test, while differences in non-parametric data were assessed by Wilcoxon's matched-pairs test or the Mann-Whitney U test. Linear regression analysis determined correlations. A $P$ value $<0.05$ indicates statistical significance.

\section{Results}

A total of 57 patients were randomized at a 1:1 ratio of once-daily dual-injection of IDeg+Lira $(\mathrm{n}=28)$ or twice-daily single-injection of IDegAsp $(\mathrm{n}=29)$. In the IDegAsp group, 28 patients completed the study, while in the IDegLira group, 24 patients completed the study. As shown in Table 1, the overall characteristics of the two treatment groups matched at baseline. The use of DPP-4 inhibitors was slightly higher in the IDegAsp group than in the IDeg+Lira group (Table 1). There were no significant differences in the incidence of diabetic complications (Table 1). 
After 52 weeks, HbA1c decreased by $0.3 \%$ in the IDegAsp group and by $0.7 \%$ in the IDeg+Lira group (Fig. 1a). Reduction in HbA1c tended to be greater in the IDeg+Lira group than in the IDegAsp group $(\mathrm{P}=0.070$; Fig. 1b). $19 \%$ of patients on IDegAsp versus $40 \%$ on IDeg+Lira achieved HbA1c $<7.0 \%$ ( $\mathrm{P}=0.1724$; Fig. 2)).

Pre-breakfast fasting blood glucose (FBG) at 52 weeks was significantly lower in the IDeg+Lira group than in the IDegAsp group $(\mathrm{P}=0.0372$; Fig. 3a), while pre-dinner $\mathrm{FBP}$ at 12 and 52 weeks was lower in the IDeg+Lira group than in the IDegAsp group (Fig. 3b).

Mean body weight decreased by $2.79 \mathrm{~kg}$ at 52 weeks from baseline in the IDeg+Lira group and by $0.78 \mathrm{~kg}$ in the IDegAsp group. Weight-loss was more common in the IDeg+Lira group than in the IDegAsp group $(\mathrm{P}=0.0638)$. Furthermore, mean BMI decreased by $1.28 \mathrm{~kg} / \mathrm{m}^{2}$ in the IDeg+Lira group and $0.32 \mathrm{~kg} / \mathrm{m}^{2}$ in the IDegAsp group (Fig. 4a). The lowering of BMIs was more significant in the IDeg+Lira group than in the IDegAsp group ( $\mathrm{P}=0.0377$; Fig. 4b).

After 52 weeks, the mean total insulin dosage was $20.5 \pm 9.7 \mathrm{U}(11.6 \pm 6.6 \mathrm{U}$ at pre-breakfast, $9.0 \pm 4.5 \mathrm{U}$ at pre-dinner) in the IDegAsp group; while, in the IDeg+Lira group, mean insulin dosages of degludec and liraglutide were $15.1 \pm 8.1 \mathrm{U}$ and $0.84 \pm 0.12 \mathrm{mg}$, respectively (Fig. 5a). Overall, the mean total insulin dose was lower in the IDeg+Lira group than in the IDegAsp group $(15.1 \pm 8.1 \mathrm{U}$ vs. $20.5 \pm 9.7 \mathrm{U}, P=0.05)$ at 52 weeks, while the mean dose of lilaglutide was $0.84 \pm 0.12 \mathrm{mg}$ at 52 weeks in the IDeg+Lira group (Fig. $5 \mathrm{~b})$.

The confirmed hypoglycemia rate was 1.32 and 0.69 per patient/year of exposure for the IDegAsp and IDeg+Lira groups, respectively. Neither the IDegAsp group nor the IDeg+Lira group experienced any episodes of nocturnal hypoglycemia.

\section{Discussion}

This is the first study to investigate the efficacy and safety of insulin degludec/insulin aspart co-formulation (IDegAsp) injected twice-daily versus the free combination of basal insulin degludec and GLP-1 receptor agonist liraglutide (IDeg+Lira) injected once-daily in patients with inadequately controlled type 2 diabetes on insulin therapy and oral anti-diabetic drugs. We demonstrated that treatment with IDeg+Lira showed no significant difference in glycemic control in reducing HbA1c when compared with IDegAsp; only a slightly better reduction in $\mathrm{HbA1c}$ at 52 weeks was observed in the IDeg+Lira group $(\mathrm{P}=0.07)$. Overall, only 19 $\%$ of patients on IDegAsp versus $40 \%$ of patients on IDeg+Lira achieved HbA1c $<7.0 \%$ after 52 weeks of treatment.

The addition of basal insulin to oral anti-diabetic agents is a well-established approach that is effective for most patients with type 2 diabetes (1). A basal-only regimen has the advantage of being well-tolerated with a lower frequency of injections and a low hypoglycemia risk, thereby facilitating patient acceptance of insulin therapy (1). Current guidelines recommend the intensification of therapy if HbA1c targets are not reached after 3-6 months of basal-only insulin therapy (7). Besides basal insulin, there are two main treatment options for intensification; the first is the addition of GLP-1RA or rapid-acting insulin analogs administered before the most substantial meal of the day, and the second option is basal-bolus insulin therapy. Alternatively, for patients on basal insulin that require additional prandial coverage, the regimen can be split into two doses of premixed insulin (1). Many patients perceive the basal-bolus regimen as being inconvenient and complicated because of both the number of daily injections and the administration of two separate types of insulins (3). The regimen of premixed insulin requires fewer injections than the basal-bolus regimen.

IDegAsp (70\% IDeg:30\% IAsp) is the first soluble combination product of basal insulin that is both an ultra-long-acting and rapid-acting insulin administered in a single injection (4). As a soluble co-formulation, IDegAsp does not require resuspension; this eases administration, and more importantly, eliminates the risk of incomplete mixing and lowers the hypoglycemia risk $(5,6)$. IDegAsp is superior in improving FPG when compared to premixed insulins, such as the biphasic insulin aspart 30 (BIAsp30); this is most likely due to differences in the extended basal components of IDegAsp, IDeg, and BIAsp30 (5, 6). A previous study 
demonstrated that IDegAsp (Ryzodeg) twice-daily is non-inferior to basal-bolus therapy in terms of HbA1c reduction (10), which indicates that IDegAsp twice-daily is a suitable alternative to basal-bolus regimens in patients with uncontrolled type 2 diabetes on basal insulin treatment.

In 2018, the ADA \& EASD consensus statement on the management of type 2 diabetes recommended GLP-RA as first-line injectable therapy, and in most cases, before basal insulin (7). The rationale for the recommendation was that the glycemic effects of GLP-1RA and insulin are similar, but GLP-1RA promotes weight loss, lowers hypoglycemia risks, and has cardiovascular benefits for patients with established cardiovascular diseases (7). Furthermore, the ADA and EASD recommended the combination of basal insulin and GLP-1RA (7). A recent study showed that IDeg+Lira provides an efficacious intensification potion with non-inferior glycemic control versus the basal-bolus in patients with type 2 diabetes on basal insulin therapy (11). Therefore, we investigated the efficacy and safety of IDegAsp co-formulation twice-daily in comparison with the free combination of IDeg+Lira once-daily in patients with inadequately controlled type 2 diabetes on insulin therapy and oral anti-diabetic drugs.

We predicted lower FBG levels in the IDegAsp twice-daily group than in the IDeg+Lira once-daily group at pre-breakfast because of its additional pre-dinner time injection. Unexpectedly, we found that pre-breakfast and pre-dinner FBG levels were significantly lower in the IDeg+Lira group than in the IDegAsp group. The reasons for lower FBG in the IDeg+Lira group remain unclear. One possible explanation is that liraglutide may act in a glucose-dependent manner to increase insulin secretions but also lower glucagon secretions post-prandially because glucagon controls plasma glucose concentrations during fasting states by increasing hepatic glucose output for circulation (12). Glucagon promotes both glycogenolysis and gluconeogenesis but inhibits both glycolysis and glycogenesis. Liraglutide in the IDeg+Lira group contributes the most to the profound reduction in FBG. In conclusion, liraglutide reduced postprandial glucose for all meals, which leads to lower fasting blood glucose levels.

In terms of the timing of daily injections, the single pre-breakfast injection of IDeg+Lira is superior to the twice-daily injections of IDegAsp at pre-breakfast and pre-dinner; the twice-daily injections are less convenient because patients perceive both the number of injection timings required and the titration needed as more burdensome (13). There is evidence of an inverse correlation between regimen complexity and patient adherence (14); this suggests that a simple treatment regimen could tackle therapeutic (clinical) inertia. As a barrier to insulin intensification, patients often cite that the burdensome regimen has a negative impact on their quality of life (15). Previous studies demonstrated that a simple titration algorithm could tackle therapeutic inertia (16). Thus, the treatment burden may be less in the IDeg+Lira group than in the IDegAsp group.

Interestingly, the prevalence of the combination with DPP-4 inhibitors in the IDegAsp group finally reached $88.5 \%$ in the present study. As a result, we also investigated the efficacy of GLP-1RA vs. DPP-4 inhibitors in patients treated with concomitant insulin injections. In general, GLP-1RAs provide superior glycemic control and weight loss vsDPP-4 inhibitors in patients with type 2 diabetes, most likely because of the supra-physiological levels of the GLP-1RA in comparison with the physiological concentrations of GLP-1 and Glucose-dependent insulinotropic polypeptide (GIP) achieved with DPP-4 inhibitors (17). However, several reports demonstrated that East-Asian people with type 2 diabetes, especially Japanese people, had a more significant reduction in HbA1c with DPP-4 inhibitors than Caucasian people (18-20). Thus, the combination therapy of insulin and DPP-4 inhibitors is a potent option for the intensification of glycemic control for Japanese people with type 2 diabetes. Our study confirmed that even in Japanese people with type 2 diabetes, the efficacy of the combination of basal insulin and GLP-1RA is superior, rather than equal, to combination of IDegAsp twice-daily and DPP-4 inhibitors.

Weight gain is always a concern when initiating insulin therapy. The 2018 ADA\&EASD consensus statement on the management of type 2 diabetes recognized GLP-RA as first-line injectable therapy before basal insulin in most cases (7). The rationale for this recommendation was the similar glycemic effects produced by GLP1RA and insulin, but GLP-1RA also induced other benefits, such as weight loss, a lower hypoglycemic risk, and cardiovascular (CV) benefits for patients with established CV diseases (7). The present study confirmed 
that the IDeg+Lira group was superior to the IDegAsp group in terms of lowering BMI or weight loss; this is likely to be due to the weight lowering effects of GLP-1RA and the insulin-sparing effects of liraglutide, which mitigates the risk of weight gain with insulin.

Since September 2019, IDegLira, which is a combination of IDeg $100 \mathrm{U} / \mathrm{mL}$ and liraglutide $3.6 \mathrm{mg} / \mathrm{mL}$, has been available in Japan: but, this ratio is based on a typical Caucasian people with type 2 diabetes. Japanese people with type 2 diabetes are more likely to have lower BMIs and endogenous insulin secretion than that of Caucasian patients. This fixed-ratio combination (FRC) may be not optimal for some Japanese patients. In the present study, we found that in the IDeg+Lira group, the mean total insulin vales for degludec and liraglutide at 52 weeks were $15.1 \mathrm{U}$ and $0.84 \mathrm{mg}$, respectively. The proportion of basal insulin to liraglutide was lower in the free combination of IDeg+Lira than the FRC of IDegLira; this ratio difference may arise from differences in the extent of obesity and/or insulin resistance between Japanese and Caucasian patients. For example, the average BMI of Japanese people s with type 2 diabetes is approximately 25, while it is approximately 32 for Caucasian people with type 2 diabetes (21). Because Japanese people with type 2 diabetes are more insulin-sensitive than Caucasians, a smaller insulin dose achieves optimum glycemic control. The fixed-ratio combination of basal insulin to GLP-1RA simplifies treatment regimens into a once-daily injection and subsequently encourages patient adherence; however, separate injections of the free combination of GLP-1RA and basal insulin may be suitable for some patients depending on individual circumstances.

The present study has some limitations. One major limitation is the small number of subjects studied. Therefore, we need to increase the number of subjects so that we do not overestimate the data. Another limitation is the open-label design, which could have affected outcomes; therefore, we cannot exclude the possibility of bias. Third limitation is that the titration of insulin dose was not performed using a predefined titration algorithm.

In conclusion, both the insulin degludec/insulin aspart co-formulation (IDegAsp) twice-daily and free combination of basal insulin degludec and GLP-1 receptor agonist liraglutide once-daily are effective intensification options for patients with inadequately controlled type 2 diabetes that are currently on insulin therapy and oral anti-diabetic drugs. This once-daily, single timing injection of IDeg+liraglutide is effective, and it is also associated with weight loss, a low hypoglycemia risk, and may overcome many barriers to treatment intensification for type 2 diabetic patients treated with basal insulin.

\section{Acknowledgments}

We thank Dr. Kunihiro Suzuki, from Oyama East Clinic, for data collection assistance.

\section{Duality of interest}

Y.A. received research funding from Nov Nordisk. Y.T., K.T., Y.C., M.M., T.J., M.S., N.F., H.K., S.M., and I.U. declare that there are no conflicts of interest regarding the publication of this paper.

\section{References}

1. Inzucchi SE, Bergenstal RM, Buse JB, Diamant M, Ferrannini E, Nauck M, Peters AL, Tsapas A, Wender R, Matthews DR; American Diabetes Association (ADA); European Association for the Study of Diabetes (EASD). Management of hyperglycemia in type 2 diabetes: a patient-centered approach: position statement of the American Diabetes Association (ADA) and the European Association for the Study of Diabetes (EASD). Diabetes Care. 2012;35(6):1364-79.

2. Moghissi E, King AB. Individualizing insulin therapy in the management of type 2 diabetes. Am J Med. 2014;127(10 Suppl):S3-10

3. Mosenzon O, Raz I. Intensification of insulin therapy for type 2 diabetic patients in primary care: basal-bolus regimen versus premix insulin analogs: when and for whom? Diabetes Care. 2013;36 Suppl 2:S212-8.

4. Heise T, Nosek L, Roepstorff C, Chenji S, Klein O, Haahr H. Distinct Prandial and Basal GlucoseLowering Effects of Insulin Degludec/Insulin Aspart (IDegAsp) at Steady State in Subjects with Type 1 Diabetes Mellitus. Diabetes Ther. 2014;5(1):255-65 
5. Fulcher GR, Christiansen JS, Bantwal G, Polaszewska-Muszynska M, Mersebach H, Andersen TH, Niskanen LK; BOOST: Intensify Premix I Investigators. Comparison of insulin degludec/insulin aspart and biphasic insulin aspart 30 in uncontrolled, insulin-treated type 2 diabetes: a phase 3a, randomized, treat-to-target trial. Diabetes Care. 2014;37(8):2084-90.

6. Kaneko S, Chow F, Choi DS, Taneda S, Hirao K, Park Y, Andersen TH, Gall MA, Christiansen JS; BOOST: Intensify All Trial Investigators. Insulin degludec/insulin aspart versus biphasic insulin aspart 30 in Asian patients with type 2 diabetes inadequately controlled on basal or pre-/self-mixed insulin: a 26-week, randomised, treat-to-target trial. Diabetes Res Clin Pract. 2015;107(1):139-47

7. Davies MJ, D'Alessio DA, Fradkin J, Kernan WN, Mathieu C, Mingrone G, Rossing P, Tsapas A, Wexler DJ, Buse JB. Management of Hyperglycemia in Type 2 Diabetes, 2018. A Consensus Report by the American Diabetes Association (ADA) and the European Association for the Study of Diabetes (EASD). Diabetes Care. 2018;41(12):2669-2701.

8. Castellana M, Cignarelli A, Brescia F, Laviola L, Giorgino F. GLP-1 receptor agonist added to insulin versus basal-plus or basal-bolus insulin therapy in type 2 diabetes: A systematic review and metaanalysis. Diabetes Metab Res Rev. 2019;35(1):e3082

9. Mathieu C, Rodbard HW, Cariou B, Handelsman Y, Philis-Tsimikas A, Ocampo Francisco AM, Rana A, Zinman B; BEGIN: VICTOZA ADD-ON (NN1250-3948) study group. A comparison of adding liraglutide versus a single daily dose of insulin aspart to insulin degludec in subjects with type 2 diabetes (BEGIN: VICTOZA ADD-ON). Diabetes Obes Metab. 2014;16(7):636-44

10. Rodbard HW, Cariou B, Pieber TR, Endahl LA, Zacho J, Cooper JG.Treatment intensification with an insulin degludec (IDeg)/insulin aspart (IAsp) co-formulation twice daily compared with basal IDeg and prandial IAsp in type 2 diabetes: a randomized, controlled phase III trial. Diabetes Obes Metab. 2016;18(3):274-80.

11. Billings LK, Doshi A, Gouet D, Oviedo A, Rodbard HW, Tentolouris N, Grøn R, Halladin N, Jodar E. Efficacy and Safety of IDegLira Versus Basal-Bolus Insulin Therapy in Patients With Type 2 Diabetes Uncontrolled on Metformin and Basal Insulin: VII Randomized Clinical Trial. Diabetes Care. 2018;41(5):1009-1016

12. Nauck MA, Kleine N, Orskov C, Holst JJ, Willms B, Creutzfeldt W. Normalization of fasting hyperglycaemia by exogenous glucagon-like peptide 1 (7-36 amide) in type 2 (non-insulin-dependent) diabetic patients. Diabetologia. 1993;36(8):741-4.

13. Peyrot M, Rubin RR, Kruger DF, Travis LB. Correlates of insulin injection omission. Diabetes Care. 2010;33(2):240-5.

14. Coleman CI, Limone B, Sobieraj DM, Lee S, Roberts MS, Kaur R, Alam T. Dosing frequency and medication adherence in chronic disease. J Manag Care Pharm. 2012;18(7):527-39.

15. Kunt T, Snoek FJ. Barriers to insulin initiation and intensification and how to overcome them. Int J Clin Pract Suppl. 2009;(164):6-10.

16. Davies M, Storms F, Shutler S, Bianchi-Biscay M, Gomis R; ATLANTUS Study Group. Improvement of glycemic control in subjects with poorly controlled type 2 diabetes: comparison of two treatment algorithms using insulin glargine. Diabetes Care. 2005;28(6):1282-8.

17. Brunton S. GLP-1 receptor agonists vs. DPP-4 inhibitors for type 2 diabetes: is one approach more successful or preferable than the other? Int J Clin Pract. 2014;68(5):557-67

18. Aso Y, Ozeki N, Terasawa T, Naruse R, Hara K, Suetsugu M, Takebayashi K, Shibazaki M, Haruki K, Morita K, Inukai T. Serum level of soluble CD26/dipeptidyl peptidase-4 (DPP-4) predicts the response to sitagliptin, a DPP-4 inhibitor, in patients with type 2 diabetes controlled inadequately by metformin and/or sulfonylurea. Transl Res. 2012;159(1):25-31.

19. Kim YG, Hahn S, Oh TJ, Kwak SH, Park KS, Cho YM. Differences in the glucose-lowering efficacy of dipeptidyl peptidase-4 inhibitors between Asians and non-Asians: a systematic review and metaanalysis. Diabetologia. 2013;56(4):696-708.

20. Davis TME, Mulder H, Lokhnygina Y, Aschner P, Chuang LM, Raffo Grado CA, Standl E, Peterson ED, Holman RR; TECOS Study Group. Effect of race on the glycaemic response to sitagliptin: Insights from the Trial Evaluating Cardiovascular Outcomes with Sitagliptin (TECOS). Diabetes Obes Metab. 
2018;20(6):1427-1434

21. Møller JB, Pedersen M, Tanaka H, Ohsugi M, Overgaard RV, Lynge J, Almind K, Vasconcelos NM, Poulsen P, Keller C, Ueki K, Ingwersen SH, Pedersen BK, Kadowaki T.Body composition is the main determinant for the difference in type 2 diabetes pathophysiology between Japanese and Caucasians. Diabetes Care. 2014;37(3):796-804.

\section{Figure legends}

\section{Fig. 1}

Hemoglobin A1c (HbA1c) over the treatment period (a) and changes in HbA1c from baseline to 52 weeks after treatment (b).

Fig.2

Proportion of patients achieving $\mathrm{HbA} 1 \mathrm{c}<7.0 \%$ at 24 weeks (a) and 52 weeks (b) after treatment

Fig. 3

Pre-breakfast fasting blood glucose (FBG) (a) and pre-dinner FBG over time (b). ${ }^{*} \mathrm{P}<0.05$ vs. IDegAsp co-formulation twice-daily.

Fig. 4

Body mass index (BMI) over the treatment period (a) and changes in BMI from baseline to 52 weeks after treatment (b). ${ }^{*} \mathrm{P}<0.01$ vs. baseline.

Fig. 5

Mean daily insulin dose (a) and mean daily liraglutide dose (b) over the treatment period.

\section{Hosted file}

Fig. 1.pptx available at https://authorea.com/users/308349/articles/456789-comparison-ofinsulin-degludec-ideg-insulin-aspart-iasp-co-formulation-therapy-twice-daily-with-freecombination-of-glp-1-receptor-agonist-liraglutide-plus-insulin-deguldec-in-tochigiideal-trial

\section{Hosted file}

Fig. 2.pptx available at https://authorea.com/users/308349/articles/456789-comparison-ofinsulin-degludec-ideg-insulin-aspart-iasp-co-formulation-therapy-twice-daily-with-freecombination-of-glp-1-receptor-agonist-liraglutide-plus-insulin-deguldec-in-tochigiideal-trial

\section{Hosted file}

Fig. 3.pptx available at https://authorea.com/users/308349/articles/456789-comparison-ofinsulin-degludec-ideg-insulin-aspart-iasp-co-formulation-therapy-twice-daily-with-freecombination-of-glp-1-receptor-agonist-liraglutide-plus-insulin-deguldec-in-tochigiideal-trial

\section{Hosted file}

Fig. 4.pptx available at https://authorea.com/users/308349/articles/456789-comparison-ofinsulin-degludec-ideg-insulin-aspart-iasp-co-formulation-therapy-twice-daily-with-freecombination-of-glp-1-receptor-agonist-liraglutide-plus-insulin-deguldec-in-tochigiideal-trial

\section{Hosted file}


Fig. 5.pptx available at https://authorea.com/users/308349/articles/456789-comparison-ofinsulin-degludec-ideg-insulin-aspart-iasp-co-formulation-therapy-twice-daily-with-freecombination-of-glp-1-receptor-agonist-liraglutide-plus-insulin-deguldec-in-tochigiideal-trial

\section{Hosted file}

Table 1 Characteristics of subjects studied.doc available at https://authorea.com/users/ 308349/articles/456789-comparison-of-insulin-degludec-ideg-insulin-aspart-iasp-coformulation-therapy-twice-daily-with-free-combination-of-glp-1-receptor-agonistliraglutide-plus-insulin-deguldec-in-tochigi-ideal-trial 